\title{
Arte Rupestre en el Karst de Ojo Guareña (Merindad de Sotoscueva-Burgos): Trabajos de Documentación y Estudio en «La Sala de La Fuente»
}

\author{
Juan A. Gómez-Barrera, Ana I. Ortega Martínez, Miguel A. Martín \\ Merino, José J. Fernández Moreno, Jesús del Val Recio, \\ Marcos García Díez, Francisco Ruiz García, \\ pablo Latorre González Moro y leandro Cámara Muñoz
}

\begin{abstract}
RESUMEN
La amplia nómina de autores que firman el presente artículo dan forma al equipo multidisciplinar que en 1995 inició un ambicioso proyecto, subvencionado por la Junta de Castilla y León, denominado «Trabajos de

Documentación y Estudio del Arte Rupestre en el Karst de Ojo Guareña».

El avance de sus primeros trabajos está recogido en el siguiente texto y fue presentado al Primer Congreso de Arqueología Burgalesa (1998), mas, al no haberse publicado éste, sigue aúr: inédito. Su interés, y de forma especial

la continuidad de la investigación, aconseja a los autores su edición en Espacio, Tiempo y Forma con vistas a una mayor difusión del proyecto $y$, con

ello, a un mejor conocimiento de la grafía rupestre de las galerías subterráneas del norte de la provincia de Burgos.

\section{ABSTRACT}

The wide number of researchers who sign the present article joined in the multi-discipline team that started an ambitious project, which was sponsored by the regional authority: Junta de Castilla y León, called "Records and Research on Cave Art at the karst in Ojo Guareña". The development of their first researches is shown in the present item and it was also presented in the First Burgos Archaeology Congress (1998), although it is still unavailable since it has never been published. Both, the importance of its contents and specially the continuity of the research led its authors to publish this article in Espacio, Tiempo y Forma so that the project could be widely known and, at the same time, provide a better knowledge of rock graphics in the underground galleries which can be found in the north of Burgos province.
\end{abstract}




$\begin{aligned} \text { PALABRAS CLAVE } & \text { KEY WORDS } \\ \text { Arte Rupestre, Grabados } & \text { Cave Art, Post-paleolithic Engravings, } \\ \text { Postpaleolíticos, Restitución } & \text { Photogrametric Restoration, } \\ \text { Fotogramétrica, Topografía, Sala de } & \text { Topography, Sala de La Fuente. } \\ \text { La Fuente. } & \end{aligned}$

\section{INTRODUCCIÓN}

El karst de Ojo Guareña se localiza al norte de la provincia de Burgos, en el término municipal de la Merindad de Sotoscueva. Forma parte de las estribaciones meridionales de la Cordillera Cantábrica, situándose en el sector de enlace entre dos grandes estructuras tectónicas: el anticlinorio de Santander-Bilbao y el gran sinclinal de Villarcayo. Entre ambas se localiza el sinclinal de La Mesa, que ha favorecido la instalación de un gran acuífero subterráneo en las calizas del Coniaciense Medio-Superior, alimentado, además de por la propia infiltración de las precipitaciones caídas en el exterior del karst, por los ríos Guareña y Trema; originados éstos en la vertiente meridional de los Montes del Somo, se convierten en subterráneos al atravesar las citadas calizas coniacienses. Las margas del Coniaciense Inferior y Turoniense Superior actúan como substrato impermeable de este acuífero.

El estudio del karst se inició en 1956 por el Grupo Espeleológico Edelweiss de la Excma. Diputación Provincial de Burgos y fue publicado (G.E.E., 1986) en un doble volumen monográfico en el que se incluía la topografía y descripción pormenorizada de más de 375 cavidades. Entre ellas destaca la red principal del Complejo Kárstico de Ojo Guareña, formada por el enlace de 11 cavidades, que con sus $100 \mathrm{~km}$. de desarrollo topografiados constituye la mayor red subterránea de España y una de las mayores del mundo.

Los hallazgos arqueológicos se sucedieron a partir de 1956 y pusieron de manifiesto la existencia de un completo registro que presenta una secuencia cultural que abarca desde el Paleolítico Medio hasta la Edad Media. Se ha detectado una ocupación diversificada, con zonas de hábitat, santuarios con muestras rupestres, sectores sepulcrales, huellas de pisadas e improntas que los diferentes grupos humanos dejaron al realizar sus incursiones al interior del Complejo, el esqueleto de un individuo que se perdió en una zona laberíntica y otros numerosos restos de difícil interpretación.

Son abundantes las estaciones con arte rupestre prehistórico, como la Sala de las Pinturas, Sala Cartón, Sala de la Fuente, Sala Keimada, 
Galería del Chipichondo, Galería de los Grabados, Galería Macarroni, Gran Diagonal, Cueva de San Bernabé, Cueva de Kaite, Cueva Kubía y Cueva la Mina. Existen otras de adscripción y/o cronología dudosa, caracterizadas por la presencia de tizonazos, como son la Galería del Sueño, Balcón de la Granja, Vía Seca, Nuevo Paso, Balcón de la Galería Principal y Museo de Cera. La necesidad de investigaciones sistemáticas propició el inicio, en 1995, del proyecto denominado Trabajos de Documentación y Estudio del Arte Rupestre en el Karst de Ojo Guareña, codirigido por Juan Antonio Gómez-Barrera y Ana Isabel Ortega Martínez y financiado por la Dirección General de Patrimonio y Promoción Cultural de la Consejería de Educación y Cultura de la Junta de Castilla y León.

Las actuaciones emprendidas hasta el momento se han centrado en la revisión de los materiales arqueológicos depositados en el Museo de Burgos y en el estudio de la Sala de la Fuente, santuario ubicado a unos $325 \mathrm{~m}$ al $\mathrm{E}$ de Cueva Palomera, desarrollado en niveles superiores a la Galería Principal.

En el nivel inferior aparecen numerosos grabados finos y multitud de tizonazos. En el nivel superior establecemos una división espacial: en el primer tercio de la galería aparecen tizonazos; el segundo se caracteriza por grabados incisos finos con representaciones figurativas esquemáticas (zoomorfos y antropomorfos) y signos (zig-zags, retículas...); y el último destaca por la profusión de grabados del tipo «macarroni», con predominio de líneas sinuosas envolventes (grandes zig-zags), sobre el que se desarrollan motivos cerrados, antropomorfos, trazos aislados y otras grafías. Esta galería también presenta un escalonamiento artificial del suelo y varios silos. En el curso de los trabajos desarrollados en 1998 se han localizado nuevas evidencias arqueológicas que amplían el repertorio artístico y material.

El trabajo acometido en la Sala de la Fuente ha consistido en la realización de un levantamiento fotogramétrico del techo y paredes de la galería superior, descripción formal y técnica de las grafías, documentación fotográfica, recogida de muestras de carbón para geocronologías e identificación taxonómica, levantamiento topográfico del conjunto, recogida y estudio de materiales superficiales.

\section{CARACTERÍSTICAS GEOMORFOLÓGICAS DE LA SALA DE LA FUENTE}

La Sala de la Fuente se localiza en el Sector Central del Complejo Kárstico de Ojo Guareña, a $370 \mathrm{~m}$ de la entrada principal de Cueva 
Palomera. Desde aquí deben descenderse los $190 \mathrm{~m}$ de trazado subhorizontal de la Rampa de Palomera, hasta enlazar con la Galería Principal y recorrer por ésta, hacia el $\mathrm{E}$, otros $180 \mathrm{~m}$ de desarrollo prácticamente horizontal.

En este punto, tras superar un tramo de reducida altura, fácilmente sifonable, la Galería Principal adquiere de nuevo sus amplias proporciones habituales, mientras que, al $S$ de un bello gours, una acusada rampa de bloques nos anuncia la existencia de un gran anchurón que se localiza en un nivel superior, cuya bóveda se eleva hasta casi $30 \mathrm{~m}$ de altura y que se conoce como la Sala de la Fuente.

Las coordenadas U.T.M. de su punto central son X: 446.790; Y: 4.764.605; Z: 673 m.s.n.m. Su piso se encuentra a $78 \mathrm{~m}$ por debajo de la entrada más elevada de Ojo Guareña y a $43 \mathrm{~m}$ por debajo del nivel de la propia entrada de Cueva Palomera.

Su génesis se vio propiciada por la confluencia de tres galerías procedentes del NW, dos de ellas pertenecientes a un nivel superior a la Galería Principal, originadas en el eje semicolmatado Galerías Altas de PalomeraBalcón de La Granja, del que distan apenas $80 \mathrm{~m}$, mientras que la tercera se debe al paso sifonable citado de la propia Galería Principal, que obligó a las aguas a excavar un nuevo conducto en la bóveda que permitiese superar el citado estrechamiento, favoreciendo el drenaje hacia el E.

En esta zona de confluencia de los tres conductos, la disolución bajo condiciones freáticas se vio facilitada por la presencia de una falla de dirección SW-NE, cuyas estrías y relleno son bien visibles junto a la pared sur. Esta discontinuidad estructural de la roca favoreció localmente la disolución de la bóveda, al remontar las aguas en busca de su nivel de equilibrio, gracias a la liberación del gradiente hidráulico. De esta forma, sobre la dirección citada se formó un gran volumen de $65 \mathrm{~m}$ de longitud proyectada, con anchura media de $15 \mathrm{~m}$ y altura de unos $25 \mathrm{~m}$ en su tramo central.

El piso de la Sala de la Fuente presenta rellenos arcillosos, arenosos y clásticos. La rampa de acceso a la sala desde la Galería Principal se ve fácilmente sometida a periódicas avenidas de agua, en época de lluvias.

En el rincón NW de la sala, a ras del suelo, incide la galería citada cuyo origen está claramente determinado por el mencionado paso sifonante de la Galería Principal, que provoca el rápido embalsamiento de agua y consiguiente sifonamiento, incluso en nuestros días, de buena parte del eje principal de Ojo Guareña. Las aguas se abrieron paso, en la bóveda, a $12 \mathrm{~m}$ de altura, agrandando por disolución un conducto de unos $3 \mathrm{~m}$ de diámetro que serpentea durante casi $40 \mathrm{~m}$ hasta desembocar en la 
Sala de la Fuente. Ésta debe precisamente su nombre al profundo gours que ocupa la mayor parte de esta galería, en cuya superficie se acumula una gruesa capa de calcita flotante, debida a la extrema sobresaturación de sus aguas. En las proximidades del gours el suelo se encuentra concrecionado. En el rincón SW se abre una corta galería que finalmente se hace impenetrable.

Sobre la pared norte de esta sala inciden, en sentido NW-SE, las otras dos galerías citadas, que se abren a $6 \mathrm{~m}$ sobre el nivel del suelo. La principal es un conducto de $36 \mathrm{~m}$ de desarrollo, por unos $7 \mathrm{~m}$ de anchura media y $3 \mathrm{~m}$ de altura, ligeramente ascendente (en sentido de la exploración), cuyo piso está recubierto de sedimentos arcillosos que casi llegan a colmatarla en su extremo NW, haciéndola impenetrable (una desobstrucción permitió la progresión durante otros $11 \mathrm{~m}$ ). La extrema sequedad de la parte final de la galería ha favorecido la aparición de cristalizaciones de yeso, tanto bajo la forma de diminutas flores de yeso fibroso, como de costras parietales a modo de grandes escamas. Paralela a ella evoluciona la otra galería, de apenas $7 \mathrm{~m}$ de recorrido penetrable y sección inferior al metro.

En la pared sur de la Sala de la Fuente, frente a los conductos citados, existen otras dos pequeñas galerías colgadas, igualmente de difícil acceso. Ambas terminan por hacerse impenetrables, aunque una de ellas permite la comunicación visual con la galería que se origina en el extremo SW del nivel inferior.

En la mitad NE de la sala se abre, a más de $25 \mathrm{~m}$ de altura, una galería colgada, sólo accesible mediante técnicas de escalada artificial, en la que la infiltración ha favorecido la litogénesis, formándose columnas estalagmíticas de hasta $8 \mathrm{~m}$ de altura, un bello gours y una gran colada pavimentaria que recubre toda la rampa de acceso a este nivel.

\section{DESCUBRIMIENTO Y PRIMERAS REFERENCIAS BIBLIOGRÁFICAS DE LA SALA DE LA FUENTE}

Los paneles de grabados localizados en las proximidades del gours, así como los abundantes tizonazos y otros restos arqueológicos, todos ellos del nivel inferior, fueron descubiertos por el Grupo Espeleológico Edelweiss en 1958, apareciendo diversas referencias en la prensa local (Diario de Burgos, 6/04/58) y nacional.

La primera cita bibliográfica de sus manifestaciones artísticas data de 1960, cuando Osaba y Ruiz de Erenchun dio cuenta de grabados fálicos y 
de las pinturas de una cabeza de cierva, un bisonte y un "cazador», supuestamente configurados a partir de múltiples trazos negros realizados con tizones, que catalogó como auriñacienses o magdalenienses (Osaba, 1960: 182, Figs. 8, 9, 10 y 11).

En 1968 el Grupo Espeleológico Edelweiss se percata de la existencia de una pronunciada rampa que permite acceder a una galería superior en la que aparece el santuario principal, informando ampliamente en la prensa local (Diario de Burgos, 30/10/68).

Ese mismo año Jordá Cerdá estudió el nivel superior, observando dos tipos de grabados: los de trazo fino con incisión aguda y otros de trazo más ancho, espatulados, con representaciones de zig-zags, líneas paralelas y sinuosas, rectángulo rayado, un barquiforme y otra figura cerrada con tres haces de líneas paralelas en su interior (Jordá, 1968-69: 68, Lám. VII, 2; VIII, 1 y VIII, 2).

En 1973, Guerra Gómez (1973), describió los grabados localizados en 1958 en las proximidades del gours, como de trazo fino e inciso con retículas, zig-zags y otras líneas quebradas de mayor grosor en su diseño.

Todo el conjunto fue clasificado por Moure Romanillo (1985: 110), mediante una referencia breve y confusa, como perteneciente al arte esquemático, situándolo cronológicamente en el Bronce Final.

El trabajo de inventario y prospección de todo el Complejo de Ojo Guareña realizado por A. I. Ortega y M. A. Martín (1986: 351-357), no pudo confirmar la existencia de los grabados y pinturas del primer nivel publicados por Osaba, al no observar parecido formal ni figurativo entre los tizonazos existentes y los calcos publicados. No obstante, se estableció una distribución espacial y temática del arte rupestre de la sala.

Tampoco han faltado diferentes referencias en obras de síntesis como son las de Bécares (1987), Gómez-Barrera (1992 y 1993), Arnáiz, Rodríguez y Rojo (1993) y Fernández y Romero (1994).

Los estudios que desde 1995 estamos llevando a cabo han propiciado la aparición de nuevos motivos artísticos y de evidencias materiales.

\section{DESCRIPCIÓN DE LAS EVIDENCIAS ARTÍSTICAS Y MATERIALES DE LA SALA DE LA FUENTE}

En el nivel inferior de la Sala de la Fuente se observan restos faunísticos, cerámicos y líticos, especialmente en su mitad SW. En la rampa de acceso desde la Galería Principal los restos son menos frecuentes debido 
a la acción erosiva de las periódicas inundaciones a que se ve sometida. En las paredes y techos bajos, especialmente en las aristas, se observan numerosos tizonazos y nódulos de sílex «in matrix», bastante deshidratados, que presentan extracciones presumiblemente de época prehistórica. En el fondo del gours, localizado en un rincón al NW de la sala, se observan abundantes restos óseos de fauna.

En torno al gours se localizan dos paneles de grabados incisos, muy finos, los únicos existentes en el nivel inferior. La temática preferente son pequeños motivos, principalmente zig-zags, si bien también pueden señalarse parrillas, retículas, un pectiforme y una gran cantidad de trazos que no concretan morfología alguna. Estas grafías no guardan similitud en el tamaño y en la ejecución con los que posteriormente describiremos en el nivel superior. Técnicamente, y de modo exclusivo, han sido observados surcos de poca anchura, escasa profundidad y sección en « $V$ ", que manifiestan el uso de un instrumental lítico de filo diedro, que bien pudiera referirse a un buril.

En el nivel superior se localiza la galería más conocida y representativa de la Sala de la Fuente. Su piso arcilloso presenta un escalonamiento artificial en el que se diferencian trece aterrazamientos, realizados con gruesos palos, observándose las marcas de sus estrías en algunos de los escalones. También existen cuatro silos realizados de manera similar a las anteriores estructuras, cuyo diámetro máximo y profundidad no supera el metro; encontránciose en el fondo de algunos de ellos restos cerámicos (Jordá, 1968-69: 68). Por toda la superficie se han encontrado restos faunísticos, un sílex, fragmentos cerámicos y abundantes restos de madera, algunos de ellos quemados.

En las paredes y bóveda de esta galería se observan numerosos grabados y trazos realizados con carbón, que hemos agrupado en catorce paneles. Para su descripción hemos realizado una división de la misma en tres sectores. En el primer tercio de la galería se sitúan una gran abundancia de trazos en carbón. A falta de un estudio definitivo de estos elementos, se apunta la posibilidad de que algunos puedan ser valorados dentro de un carácter intencional y no como meras improntas producto del reavivado de teas.

En el segundo tercio se concentran grabados de trazo fino e incisos. Entre ellos destacan varios antropomorfos esquemáticos (Ortega y Martín, 1986: 352-353) de los que tres, en trazo muy fino, muestran cuerpos largos, piernas cortas y brazos abiertos. Un cuarto se diseña con brazos en cruz y trazo axial bifurcado para dibujar las piernas, mientras que la cabeza se forma aprovechando el resalte de un fósil de morfología redon- 
deada y la mitad inferior del cuerpo queda enmarcada trazando un faldellín que recuerda, lejanamente, a los antropomorfos de la Galería del Sillex de Atapuerca (Apellániz y Uribarri, 1976). Un quinto antropomorfo, también esquemático, aparece asociado a un cérvido de amplia cornamenta y numerosos candiles (Lám. I), por la técnica de raspado empleada y su forma, recuerda a los vistos en la Cueva de Kaite (Ortega y Martín 1986: 347 y 352).

Junto a estos motivos surgen otras figuras zoomorfas (Lám. II), muy características por su técnica de raspado, tronco largo y ancho y extremidades, cola y cuernas - sin candiles - largas y de trazo fino. Son también abundantes en este sector las retículas (Lám. III), agrupaciones de zigzags paralelos y otros motivos.

Los modos técnicos observados son variados: instrumental de materia vegetal, pétreo con bisel en la parte activa y un tercero de morfología redondeada. Los primeros, a modo de arrastre y realizados con un palo o rama, presentan un fondo de surco con estrías internas producto de la acción erosiva. La anchura es variable entre 3 y $7 \mathrm{~mm}$. La sección del surco se presenta generalmente cóncava, a lo que cabría matizar que en algunos de los casos el fondo de la misma ofrece una sinuosidad variable producto de la diferente profundidad que alcance cada una de las estrías generadas.

La utilización de instrumentos pétreos con bisel presenta anchuras variables entre 2,5 y $5 \mathrm{~mm}$, encontrándose en relación directa con la amplitud de la parte del útil que entró en contacto con el soporte. Dependiendo de la disposición del bisel respecto al soporte la sección del surco será en « $V$ », simétrica o asimétrica o en «U», percibiéndose en algunos de los efectivos en el fondo del surco, microestrías producidas por la morfología de la parte activa del útil o por el arrastre y acción erosiva de algún grano asociado a la arcilla de descalcificación o bien por ambas cosas a la vez.

El uso de instrumental de morfología redondeada muestra una sección con morfología en «U» y profundidad marcada. Las anchuras de los trazos varían entre 3 y $6 \mathrm{~mm}$.

El último tercio de la galería ofrece la presencia de un conjunto de grabados cuyo tema central, un gran zig-zag envolvente (Lám. IV), fue realizado con los dedos, ocupa buena parte de la bóveda. A él se asocian retículas, trazos paralelos, diversos zig-zags, un emparrillado cuadrangular y varias figuras cerradas, una de las cuales ha sido comparada con los ídolos de la Edad del Bronce (Ortega y Martín, 1986: 355) (Lám. V). También se ha destacado (Ortega y Martín, 1986: 355-356) una figura (Lám. VI) con evidentes similitudes con los dos grandes antropomorfos de la Sala de 
Arte Rupestre en el Karst de Ojo Guareña (Merindad de Sotoscueva-Burgos)...
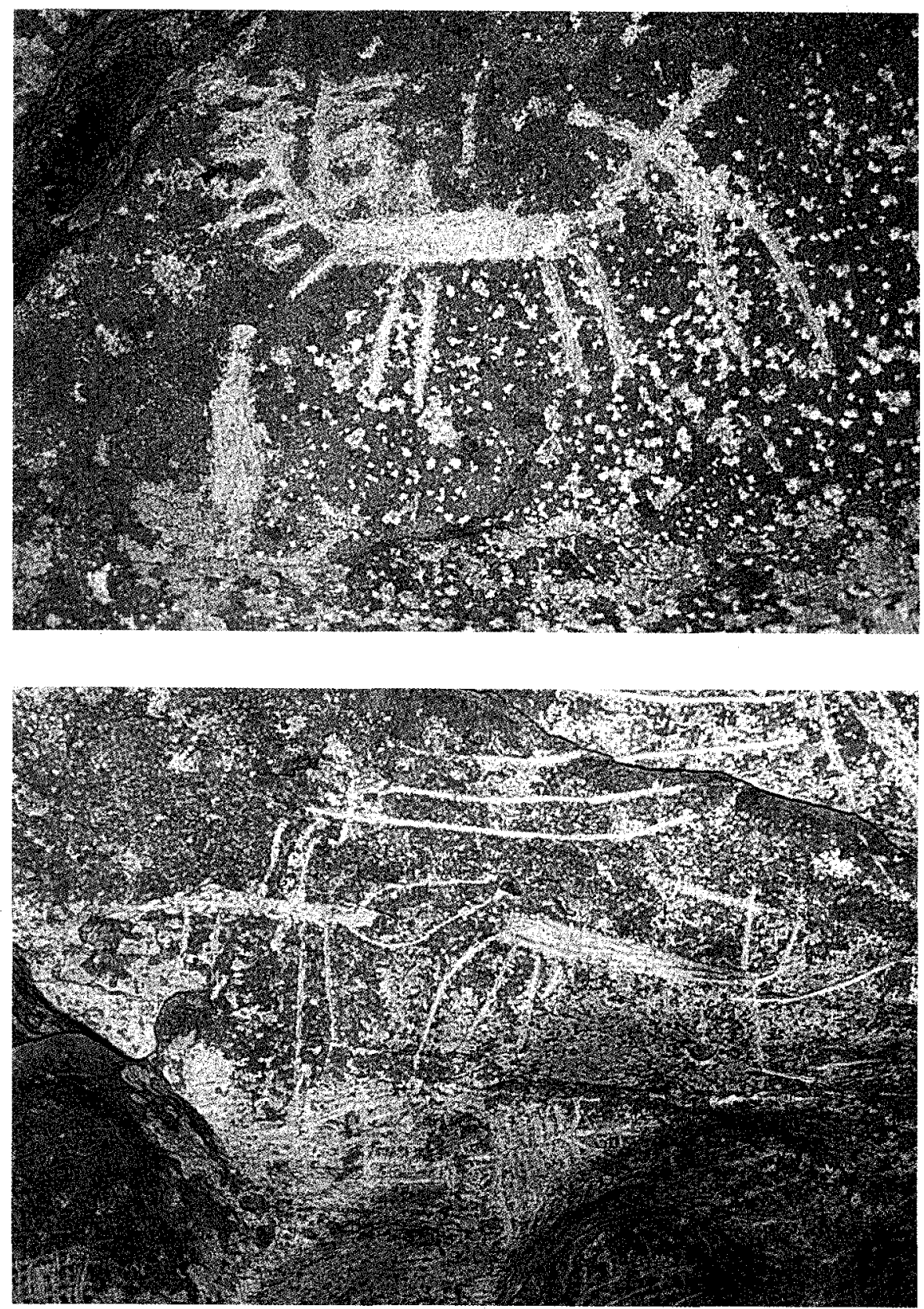

Lám. I y II. Antropomorfo asociado a representación zoomorfa (cérvido) y representaciones zoomorfas (Foto M. A. Martín Merino). 

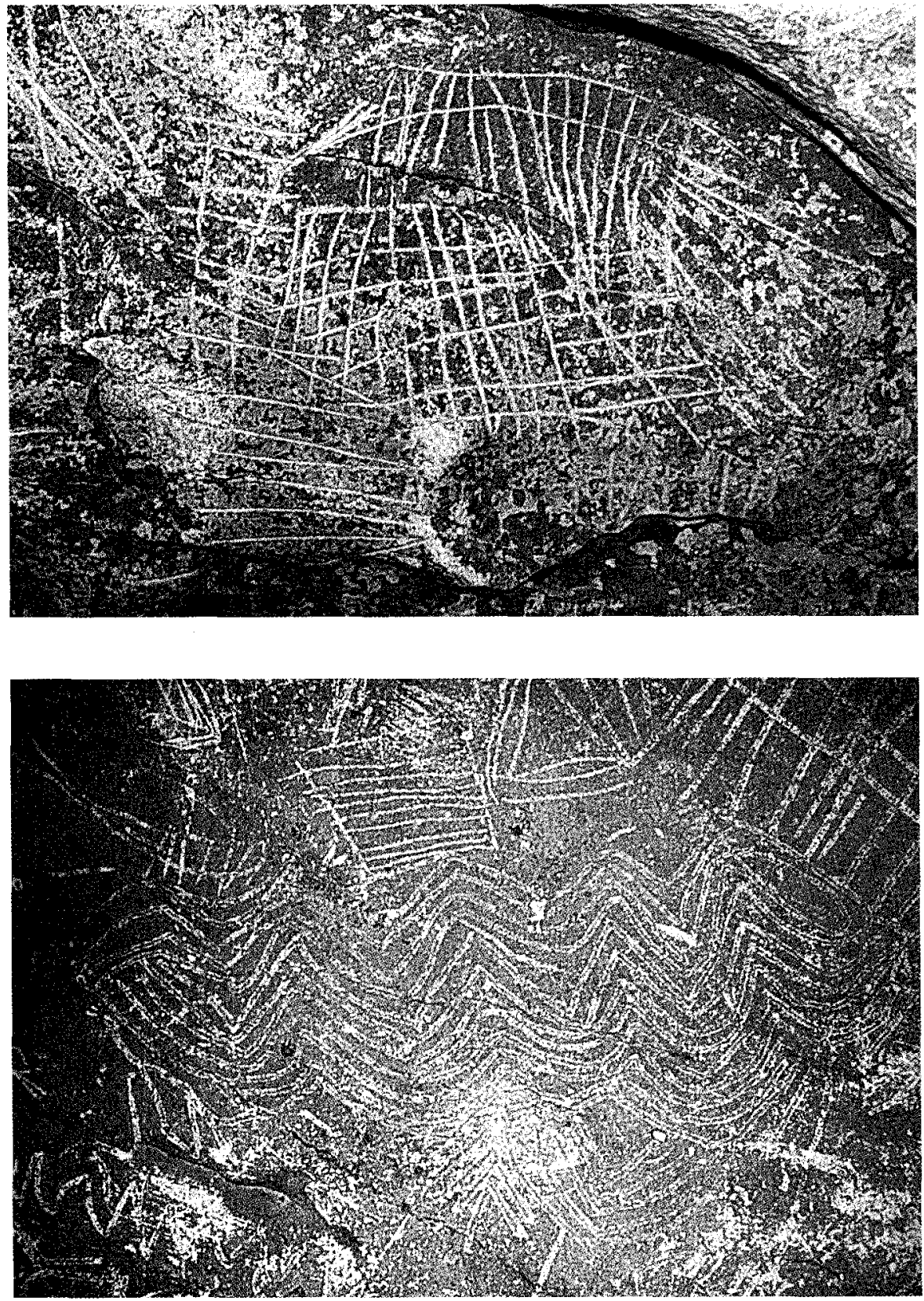

Lám. III y IV. Motivos reticulados, escaleriformes y trazos paralelos arriba $y$, abajo, gran zigzag envolvente asociado a otros temas (Foto M. A. Martín Merino). 
Arte Rupestre en el Karst de Ojo Guareña (Merindad de Sotoscueva-Burgos)...

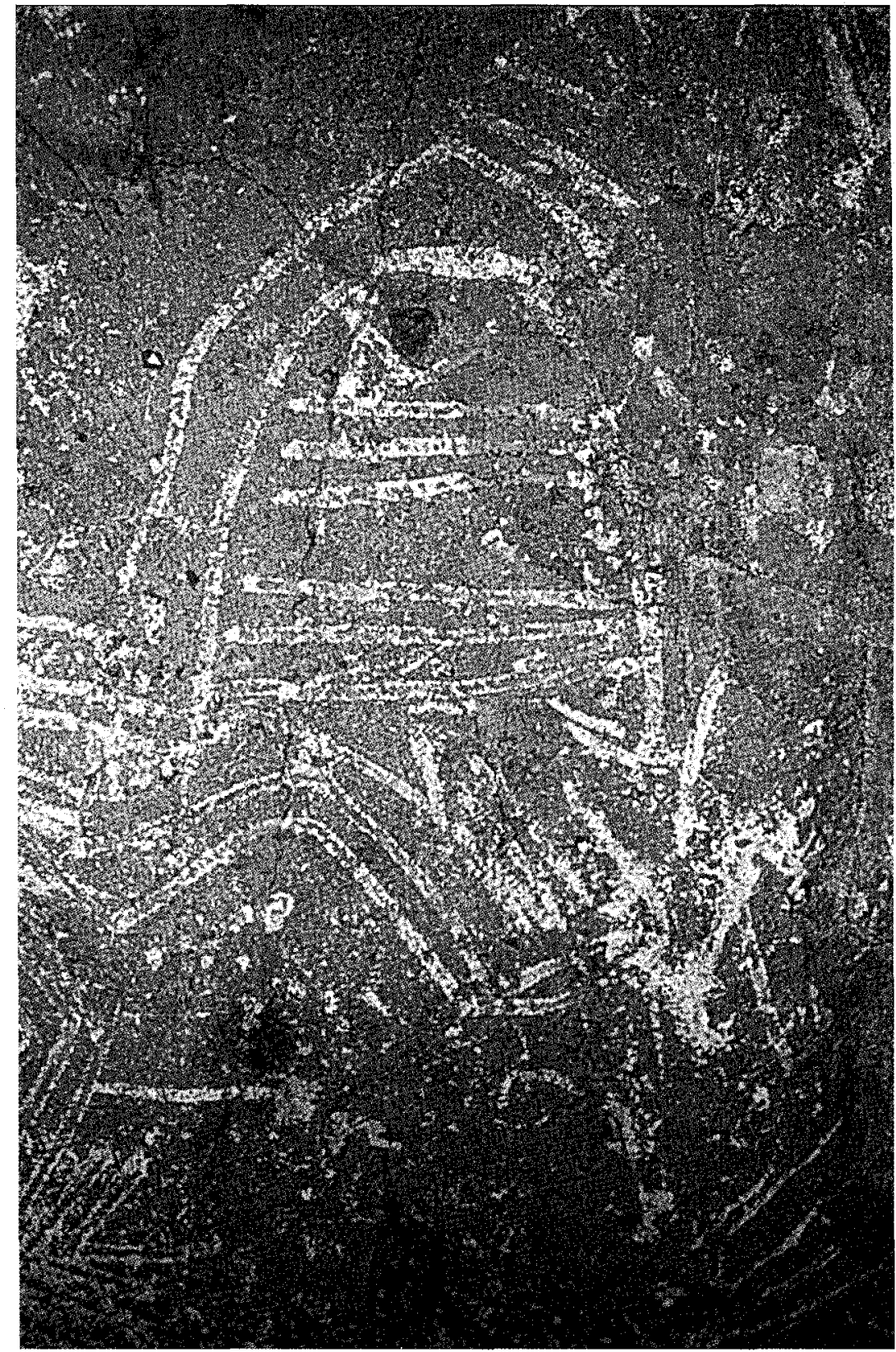

Lám. V. Figura cerrada similar a algunos ídolos de la Edad del Bronce (Foto M. A. Martín Merino). 


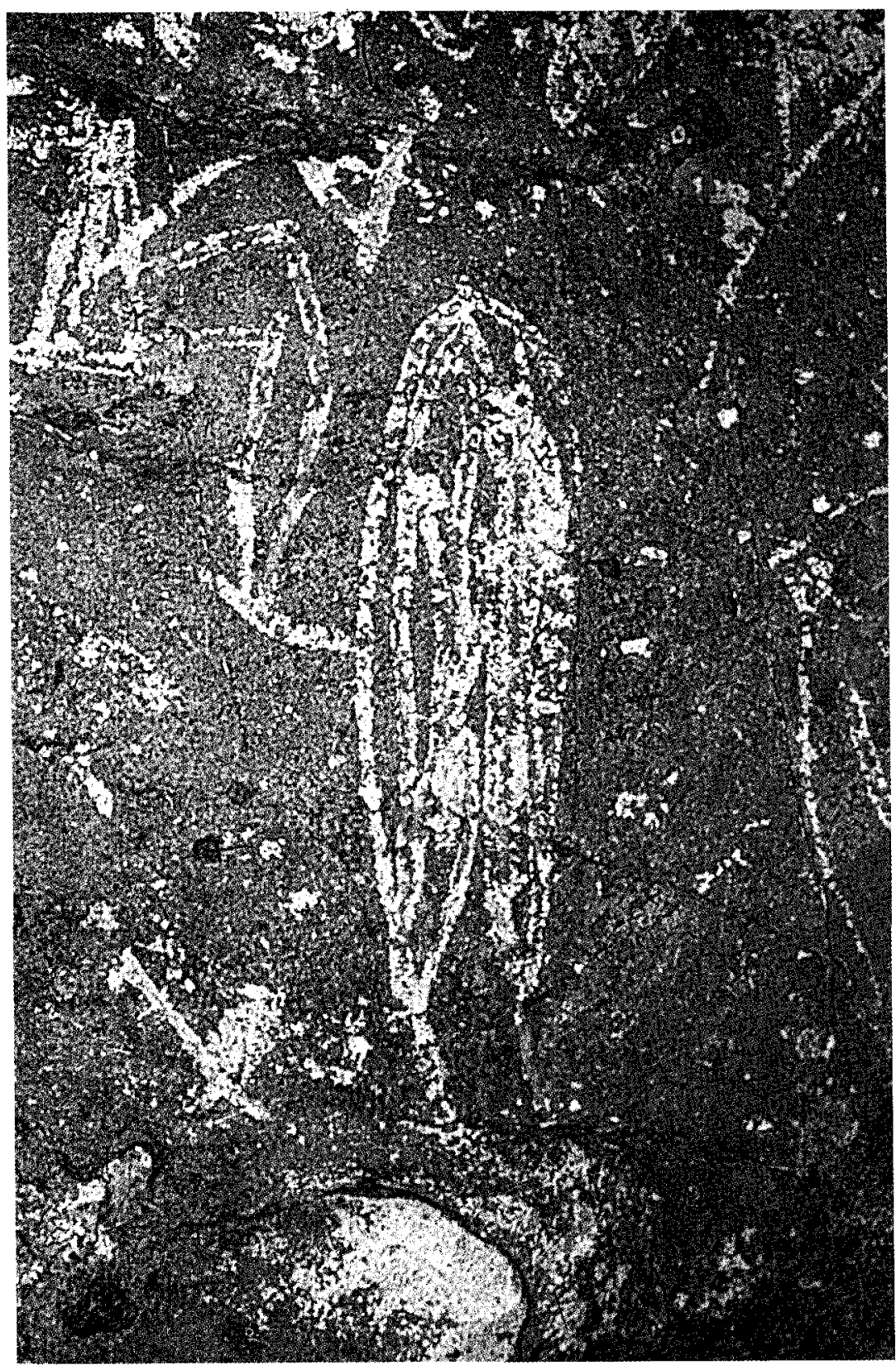

Lám. VI. Posible antropomorfo de trazado parecido a los de la Sala de las Pinturas (Foto $M$. A. Martín Merino). 
las Pinturas, uno de ellos recientemente datado en $11.540 \pm 100$ B.P. (Corchón et alii, 1996). Por último, en la pared oriental de este sector se localiza una figura de vulva y una representación descrita como la cabeza de un ciervo en trazo raspado discontinuo, que contornea un relieve natural (Ortega y Martín, 1986: 355-357).

Las maneras de grabar reconocidas son dos: en primer lugar la incisión descrita anteriormente como el resultado de la acción de un instrumental pétreo con bisel en la parte activa $y$, una nueva realizada con los dedos, o genéricamente denominada digital. Esta última, manifiesta tanto a modo de arrastre como de impresión, es la mayoritaria dentro de este sector. El arrastre digital se caracteriza por surcos de gran anchura, de 6 a $20 \mathrm{~mm}$, y secciones de morfología en "U» poco profundas. Las de impresión se reconocen por la presencia de depresiones de morfología tendente a circular, si bien también puede apreciarse algunas ligeramente elipsoides.

En la campaña de 1998 se han localizado, en este mismo nivel, tres nuevas galerías de escaso desarrollo. Una de ellas, paralela a la anterior, posee dos escalones artificiales, tizonazos y abundantes restos de madera, entre ellos un gran fragmento de unos $30 \times 30 \mathrm{~cm}$ y $5 \mathrm{~cm}$. Frente a las anteriores se sitúan las otras dos: una posee abundantes tizonazos y un panel de finos grabados, tres zig-zags incisos, cuyo surco se encuentra totalmente cristalizado por yesos; en la otra también existe una pequeña muestra de tizonazos y se ha localizado un cráneo de cánido depositado antrópicamente en una grieta.

De modo preliminar pueden establecerse un conjunto de relaciones entre técnicas, temas y tipometría. Las relaciones entre la técnica y la tipometría, dentro del nivel superior, se concretan en la separación entre motivos ejecutados con la técnica digital y el resto. A grandes rasgos, puede señalarse que aquellos realizados mediante técnica digital presentan unos tamaños sensiblemente superiores al resto de los ejecutados con otras técnicas, si se exceptúa el gran zig-zag localizado en el techo de la bóveda. En el sector intermedio se encontrarían aquellos realizados con instrumental de morfología activa en bisel y redondeada cóncava; ambos, y especialmente el primero de ellos, muestran los formatos más pequeños. Los realizados mediante materia vegetal no carbonizada muestran formatos pequeños e intermedios.

Las relaciones técnicas y temáticas están lejos de ser descritas de una manera absoluta en lo que a la totalidad iconográfica se refiere, si bien creemos que pueden mencionarse una serie de apreciaciones cualitativas que muestran la tendencia general del dispositivo gráfico. Partiendo de una amplia distinción entre motivos denominados figurativos (zoomorfos y 
figuraciones humanas) y abstractos, se observa una diferencia técnica entre ellos: La práctica totalidad de los figurativos, un escaso número del total, han sido ejecutados mediante un instrumental con morfología de la parte activa biselada, llegando en algunos casos a utilizarse el instrumento no sólo para incidir, sino para crear una zona (tronco de las representaciones zoomorfas) raspada. En contraposición, dentro de los llamados abstractos se da la totalidad de los tipos técnicos. Observaciones someras vendrían a manifestar un marcado predominio de la incisión con instrumental en morfología biselada para la ejecución de las retículas y zig-zag. Motivos cerrados, exceptuando las retículas, han sido realizados preferentemente en técnica digital.

\section{RESTITUCIÓN FOTOGRAMÉTRICA DE LOS GRABADOS DE LA SALA DE LA FUENTE}

Dadas las características geomorfológicas de la Sala de la Fuente resultaba inevitable la búsqueda de un sistema de documentación gráfica indirecta que nos permitiera copiar, con absoluta fidelidad y nulo riesgo de alteración de los soportes, los distintos paneles artísticos apreciados en sus paredes y techumbre.

En este sentido, y dada la distribución de los grabados, en su mayoría ocupando la bóveda de la sala, pensamos que el método adecuado sería la restitución fotogramétrica. Ya conocíamos sus posibilidades a partir de los trabajos efectuados por A. Llanos y F.J. García Lázaro, en 1977, en el techo de la sala de los polícromos de Altamira (Llanos y García Lázaro, 1980a y 1980b).

La fotogrametría permite definir con precisión formas, dimensiones y posición en el espacio de un objeto - y por lo mismo, de una pintura o un grabado-. La UNESCO la estimó como el procedimiento más idóneo para el registro e inventario de bienes culturales. El Dpto. de Arqueología del Instituto de Conservación y Restauración de Bienes Culturales del Ministerio de Cultura realizó, desde 1982, tomas fotográficas y pares estereoscópicos, como parte del Proyecto Nacional de Arte Rupestre, en la Cueva Pintada de Galdar. San Nicolás del Toro lo aplicó directamente en las estaciones murcianas del Abrigo I del Milano (Mula), Abrigos del Pozo (Calasparra), la Cañaíca del Calar y Fuente de Sabuco (Moratalla), glosando la bondad del método, pese a su excesivo coste, y concluyendo que la obtención de pares estereoscópicos de los conjuntos de arte rupestre con apoyo topográfico y, en la medida de lo posible, su restitución, viene exigida por dos factores interrelacionados: es el único procedimiento que 
garantiza una recogida objetiva de la información y su generalización permitirá disponer de un catálogo sin los subjetivismos que lastran en la actualidad gran parte de la documentación (San Nicolás, 1989: 13-14).

Aparte las descripciones ya mencionadas, la documentación gráfica del arte rupestre de la Sala de la Fuente se limitaba a una serie de fotografías de los motivos más llamativos, por repetidos, y a unos pocos calcos trazados a mano alzada o sobre las propias fotografías, que en la mayoría de los casos se habían obtenido en condiciones precarias de iluminación y encuadre. En todo caso, los dibujos y fotografías hasta entonces realizados y publicados daban una visión descontextualizada de los paneles, sin referencia a su ubicación dentro de los espacios de la cueva, ni a las relaciones entre ellos.

La aplicación de la fotogrametría a la Sala de la Fuente se planteó como el ensayo de un método de documentación que, de resolver estos problemas, pudiera ser aplicado extensivamente al estudio documental del resto de las muestras rupestres del Complejo. Se ha ensayado su resultado por tratarse, como ya se ha indicado, de una pequeña galería de suelo arcilloso con gran pendiente, en la que se encuentra un conjunto amplio, interesante y mal conocido, de grabados efectuados sobre la sutil capa de arcilla que recubre la bóveda.

En efecto, la fotogrametría terrestre informatizada que hemos aplicado tiene para el caso una serie de ventajas sobre otros medios de documentación que la hacen teóricamente idónea, a saber:

a) La toma de fotogramas dentro de la sala se pudo efectuar en unos pocos días, evitando así el tedioso e incómodo trabajo de dibujo «in situ».

b) Los motivos decorados no fueron tocados en ningún momento para obtener su dibujo, evitando el riesgo que conllevaría la realización de calcos directos, dado que la arcilla sobre la que se diseñan se encuentra aún fresca.

c) La fotogrametría informatizada da como resultado modelos digitales tridimensionales, con lo que se obtiene una representación de los grabados no como forma plana sino con el relieve propio del substrato rocoso en que se encuentran. Además, es posible obtener una representación de la forma del contenedor - la galería- y dar una idea más real del espacio en que se encuentran los paneles.

d) El apoyo topográfico efectuado permite operar con distintos modelos fotogramétricos -es decir, con múltiples fotos tomadas desde distintas posiciones y con orientaciones apropiadas para la representación posterior del objeto-y situar el resultado de la restitución de todas ellas 
en un solo sistema de coordenadas tridimensionales. De este modo se puede también apreciar la disposición relativa de los distintos paneles y evaluar el significado que tales relaciones pueden tener.

e) Evaluadas estas relaciones y discriminados los motivos que incorporan los paneles, es posible, gracias a la estructuración en «capas» que permite la aplicación de CAD empleada, dar representaciones analíticas, secuenciales, etc. del modelo.

f) Además, dentro del campo de las aplicaciones informáticas, se puede desarrollar un sistema de información que relacione el resultado gráfico de la investigación -el modelo digital de las distintas salas del Complejo- con una base de datos que contuviera toda clase de información relacionada. Un sistema de consultas basado en SQL permitiría adelantar en la investigación al establecer relaciones objetivas entre los distintos elementos del sistema.

Teniendo en cuenta cuanto queda dicho, los trabajos efectuados en la restitución fotogramétrica de los grabados de la Sala de la Fuente se llevaron a cabo en un triple nivel que permitió una toma de datos, la restitución de los mismos y la manipulación del modelo y edición de planos. La toma de datos se pudo realizar gracias al empleo de los siguientes medios:

- Cámara fotográfica semimétrica Hasselblad SWC/M de $38 \mathrm{~mm}$ de focal, sobre diapositiva Fuji Velvia de 50 ASA y Provia de 100 ASA, de 6x6 $\mathrm{cm}$ de formato.

- Dos estaciones totales Pentax PTS 20F de 20 seg. de precisión angular con distanciómetro infrarrojo de $0,5 \mathrm{~cm}$ de precisión con miniprisma especial para trabajos de distancias próximas.

- Cámara fotográfica Bronica de $40 \mathrm{~mm}$ de focal sobre película polaroid de $6 \times 6 \mathrm{~cm}$, para la realización de pruebas de iluminación con flash previas a la toma de los fotogramas y asegurar la corrección de éstos en cuanto a definición de imagen, profundidad de campo, etc.

- Cámaras fotográficas de formato convencional para la documentación general del proceso de trabajo.

- Señales luminosas de pequeño tamaño e intensidad y dispositivo de emisión láser, para la obtención de perfiles de distintas secciones de la sala por fotogrametría estereoscópica.

Se obtuvieron un total de cuarenta y seis pares de fotogramas para la documentación de los parámetros de la galería, tres pares de fotogramas para la obtención de perfiles por rayo láser y, para el apoyo topográfico, 
sesenta y siete lecturas desde la estación total, sobre un conjunto de cuarenta y dos señales adhesivas fijadas a la superficie de la cueva.

La restitución fue efectuada por una persona, arqueóloga con formación de operadora de restitución, con el siguiente equipo:

- Estereorrestituidor analítico Adam MPS2 de 5 micras de precisión, con marca flotante de 20 micras de diámetro, óptica de hasta 35 aumentos para diapositiva o negativo de $6 \times 6 \mathrm{~cm}$.

- Ordenador personal Tandon $486 \mathrm{DX} 2 / 50$, con $20 \mathrm{Mb}$ de RAM y 100 $\mathrm{Mb}$ de disco duro, tarjeta de vídeo de alta resolución y monitor Sony de 14 ".

- Programas específicos de orientación de modelos fotogramétricos de Adam System 2.60, interfaz de Adam con Autocad y programa de diseño asistido Autocad versión 14.

$Y$, finalmente, la manipulación del modelo y edición de planos se llevó a cabo con el equipo:

- Ordenador personal DFI 486 DX2/66 con 32 MB de RAM y 1 Gb de disco duro, tarjeta de vídeo Vesa de alta resolución y monitor NEC MultiSync 6FG de 21 ".

- Ordenador personal Pentium a $200 \mathrm{mhz}$ con $64 \mathrm{mb}$ de RAM y sistema operativo Windows NT 4.0.

- Impresora de inyección de tinta en color Epson de 1440 p.p.p.

- Programa Autocad versión 14.

El resultado del trabajo se concreta en un conjunto de vistas del modelo tridimensional (Fig. 1 y 2) de la Sala de la Fuente, planta, secciones, perspectivas y detalles, obtenidas mediante el programa de CAD empleado. Acompañando a éste se presenta el modelo digital tridimensional de la sala en formato informático tipo DWG. Sin embargo, la verdadera documentación completa de la Sala de la Fuente está formada por la colección de fotogramas ordenados en pares estereoscópicos con su apoyo topográfico. Es en estas fotos - que en su día se entregarán a la Administración como parte de nuestro trabajo- donde se encuentra enteramente reflejado el estado de la sala en el momento de la documentación, y hay que entender que el modelo digital restituido es sólo una selección de la información allí contenida con el objetivo concreto de dibujar los paneles y dar una idea del espacio en que se encuentran y de las relaciones que hay entre ellos.

El trabajo de restitución fotogramétrica de la Sala de la Fuente, tal y como aquí se expone, es el resultado de dos campañas de toma de datos 


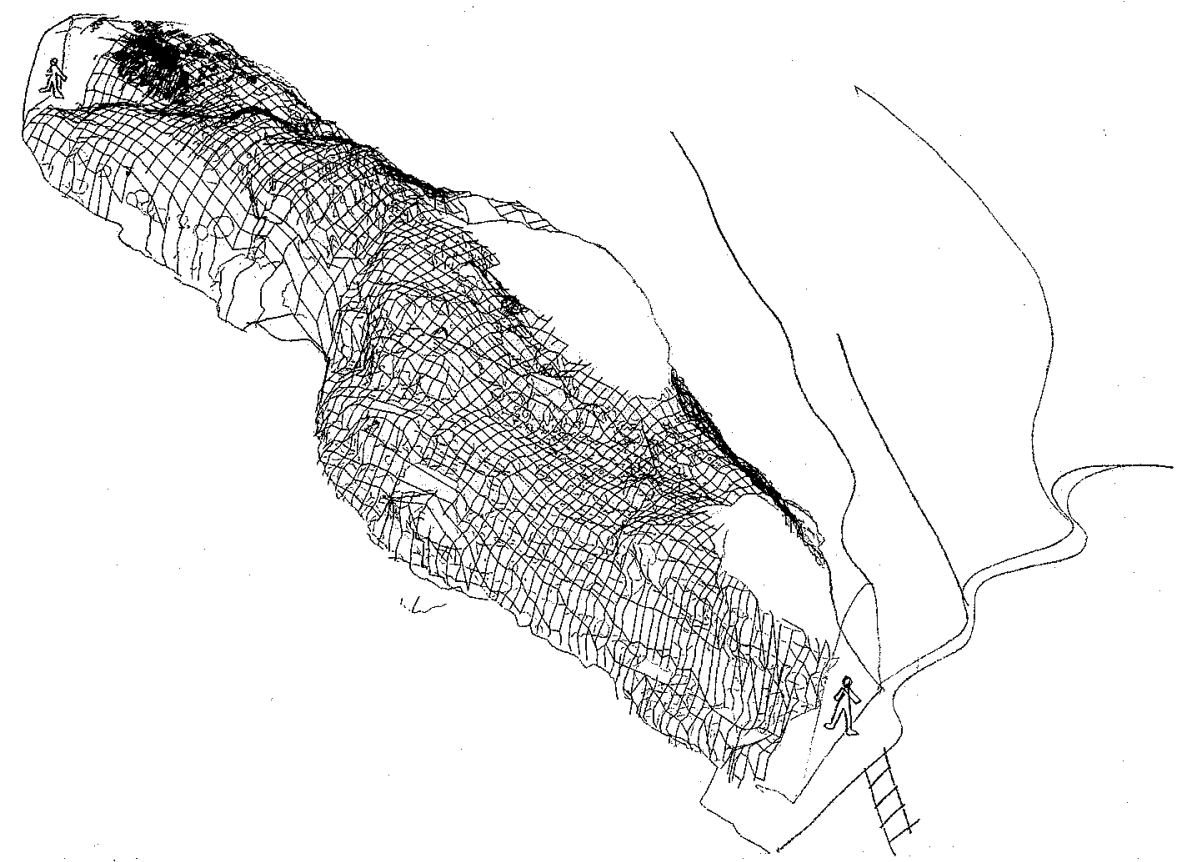

Fig. 1. Restitución fotogramétrica y croquis visual del nivel superior de la Sala de la Fuente, según Latorre y Cámara.

y de su comprobación por los arqueólogos en laboratorio e «in situ», determinando aquellas correcciones necesarias.

Hay que hacer notar que las incorrecciones apreciadas al método son debidas, en parte, a las peculiaridades de esta galería, caracterizada por la irregular topografía de bóveda y paredes, que provoca tomas fotográficas muy forzadas que distorsionan ciertos motivos. A su vez deben añadirse la abundancia de elementos naturales (fisuras, venas de calcita, estilolitos y desconchados) que enmarañan el conjunto y se confunden, a veces, con los propios trazos antrópicos o grabados. No obstante, algunos de estos elementos, especialmente las fisuras, pueden ser representados al compartimentar el substrato rocoso en el que, en ocasiones, los motivos grabados parecen estar encuadrados por estas morfologías naturales.

Aparte de las dificultades de origen natural citadas, se añaden otras inherentes a los propios grabados: surcos poco marcados y con escaso resalte sobre la fina capa de arcilla que recubre la bóveda y paredes, lo 


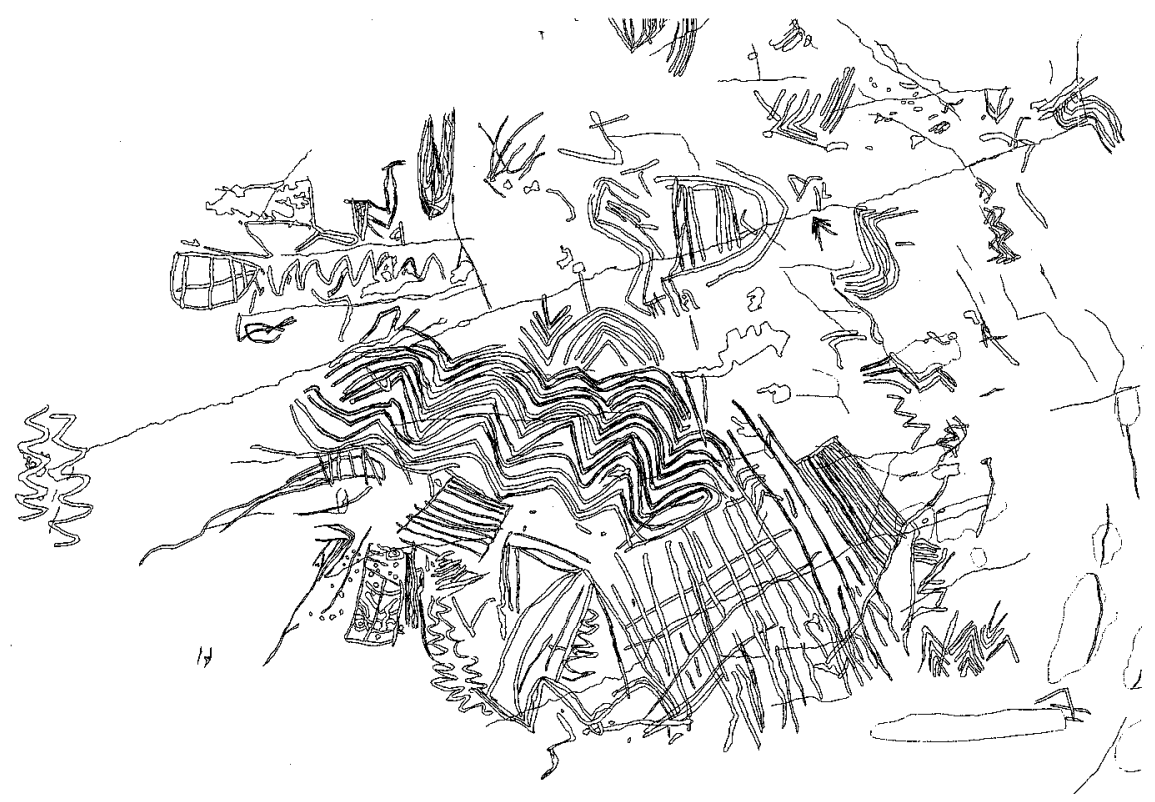

Fig. 2. Detalle de la restitución fotogramétrica del panel principal de La Sala de la Fuente, según Latorre y Cámara.

que unido a la gran acumulación y complejidad de trazos existentes dificulta la lectura de los datos a la hora de restituir los pares estereoscópicos.

Estas dificultades observadas hacen aconsejable que la restitución sea realizada por una persona buena conocedora del espacio donde se enmarcan las evidencias artísticas y los fenómenos litológicos antes citados, para discriminar convenientemente éstos de las grafías. Aún así, para el caso concreto de la Sala de la Fuente se hace necesario complementar estos trabajos de gabinete con posteriores comprobaciones «in situ» que depuren el resultado inicial de la restitución.

\section{BARRIDO FOTOGRÁFICO DE LA SALA DE LA FUENTE}

Una parte importante de las tareas de documentación llevadas a cabo en la Sala de la Fuente se ha centrado en la obtención de una correcta, exhaustiva y completa documentación fotográfica (en blanco y negro, color y diapositivas) de todos y cada uno de los motivos grabados del recinto. 
En la campaña de 1996 se procedió a un sistemático «barrido fotográfico" del sector superior de la sala; previamente a su ejecución se comprobó el Archivo Fotográfico del Grupo Espeleológico Edelweiss y se obtuvieron de él copias del material utilizable. Estos trabajos, unidos a la restitución fotogramétrica que se poseía por entonces del yacimiento, nos permite acercarnos de forma más precisa al contenido real de la galería. La documentación obtenida en ese año constaba de 780 diapositivas, 72 fotografías en color y 26 copias en blanco y negro seleccionadas del archivo del G.E.E.

Durante la campaña de 1997 se completó el «barrido fotográfico» del nivel superior, que se amplió al sector inferior del yacimiento, obteniéndose un total de 69 diapositivas de sus paneles grabados, aparte de las realizadas para los tizonazos repartidos por toda la sala. El número de diapositivas disponibles a finales de la campaña de 1997 alcanzaba la cifra de 949 de las que se proporcionó a la Administración Autonómica una selección de 128, abarcando con ella todos los paneles artísticos de la Sala de la Fuente.

\section{TRABAJOS TOPOGRÁFICOS EN LA SALA DE LA FUENTE}

Aunque contábamos, desde 1968, con una topografía de la Sala de la Fuente levantada por el Grupo Espeleológico Edelweiss, en concreto por Pedro Plana Pañart, M. ${ }^{2}$ Carmen López Alonso y Miguel Ángel del Álamo Ortiz, los últimos trabajos efectuados en el yacimiento han estado encaminados al levantamiento de una nueva topografía de detalle, que pudiera ajustarse en mayor medida a las necesidades requeridas para el estudio del arte rupestre.

El levantamiento topográfico de la Sala de la Fuente se planteó con el fin de responder a dos objetivos prioritarios: reflejar la situación exacta de los restos arqueológicos (paneles, tizonazos, escalonamiento artificial, silos, etc.) y dar el apoyo gráfico imprescindible a la descripción morfológica, genética y su contexto.

Los trabajos de campo se iniciaron desde la gatera final del nivel superior, en dirección NW-SE, sin fijarnos unos límites precisos. Al final, y atendiendo al segundo punto de nuestros objetivos, se concluyó que era preciso incluir en la reconstrucción topográfica todos los conductos a diferente nivel que se conjugan en el gran volumen de la sala.

El método topográfico empleado ha consistido en el establecimiento de una línea poligonal de unión entre estaciones principales, desde las cuales 
se lanzaron radiales a toda la serie de puntos que definen la morfología de la sala (esquinas, contacto suelo-pared, escarpes, bloques, espeleotemas, etc.), así como a todos los lugares de interés arqueológico.

Se obtuvieron 243 puntos topográficos, los cuales quedaron convenientemente señalizados en el terreno, siendo la suma total de todas las visuales de $1.364,8 \mathrm{~m}$.

El material empleado ha estado compuesto por una brújula Suunto de precisión $0,5^{\circ}$, clinómetro Suunto de precisión $1 .^{\circ}$, cinta métrica inextensible de precisión $0,01 \mathrm{~m}$, y dos jalones para fijar las visuales entre estaciones. Un puntero láser y una escalera extensible sirvieron para la toma de medidas a puntos inaccesibles del techo.

Una vez obtenidas todas las medidas de campo, se introdujeron en el ordenador a fin de convertir las coordenadas relativas entre puntos (brújula, clinómetro, distancia) a las coordenadas U.T.M. (X, Y, Z) con las que se referencia el Complejo de Ojo Guareña, pudiendo de esta manera efectuar la planimetría (Fig. 3) y decidir cuáles serían las secciones transversales más idóneas. La escala original del dibujo es de 1:100, que permite la representación de todos los detalles, siendo la precisión obtenida del $99,64 \%$.

Con respecto a las secciones, primeramente se obtuvieron las transversales de la galería del nivel superior, trabajo que permite observar la evolución de las formas de sección del conducto, desde la colmatación final hasta su conjunción con la Sala de la Fuente. En segundo lugar, se obtuvo un perfil (Fig. 4), en realidad un abatimiento de las proyecciones de los diferentes conductos sobre un solo plano, en el que se representan los niveles que convergen en la sección transversal de la sala, representación indispensable para conocer la génesis del conducto. Por último, se realizó un perfil cuyo plano de proyección, perpendicular al anterior, nos proporciona una visión de las vastas dimensiones del conjunto, $40 \mathrm{~m}$ de longitud, $19 \mathrm{~m}$ de anchura y $25 \mathrm{~m}$ de altura, y de su situación con respecto al acceso actual desde la Galería Principal.

\section{VALORACIÓN DE LOS TRABAJOS EFECTUADOS Y PLAN DE ACTUACIÓN FUTURA}

A pesar de lo avanzado del proyecto de estudio y documentación de la Sala de la Fuente, dentro del análisis del arte rupestre de Ojo Guareña, no 
Sala de la Fuente

Sector Central - Ojo Guareña

(Merindad de Sotoscueva, Burgos)

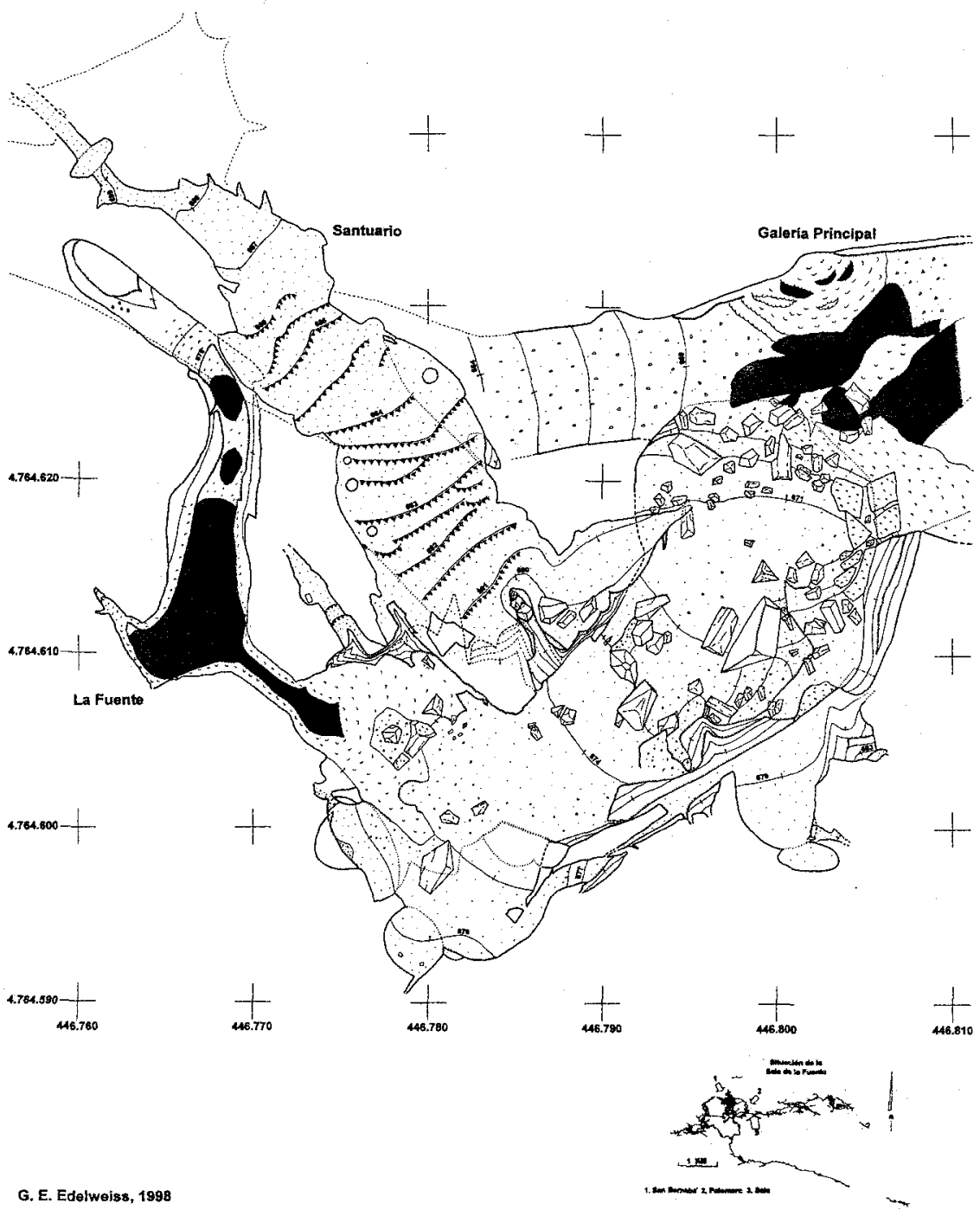

Fig. 3. Topografía (planta) de la Sala de la Fuente. 


\section{Sala de la Fuente}

Sector Central - Ojo Guareña

(Merindad de Sotoscueva, Burgos)

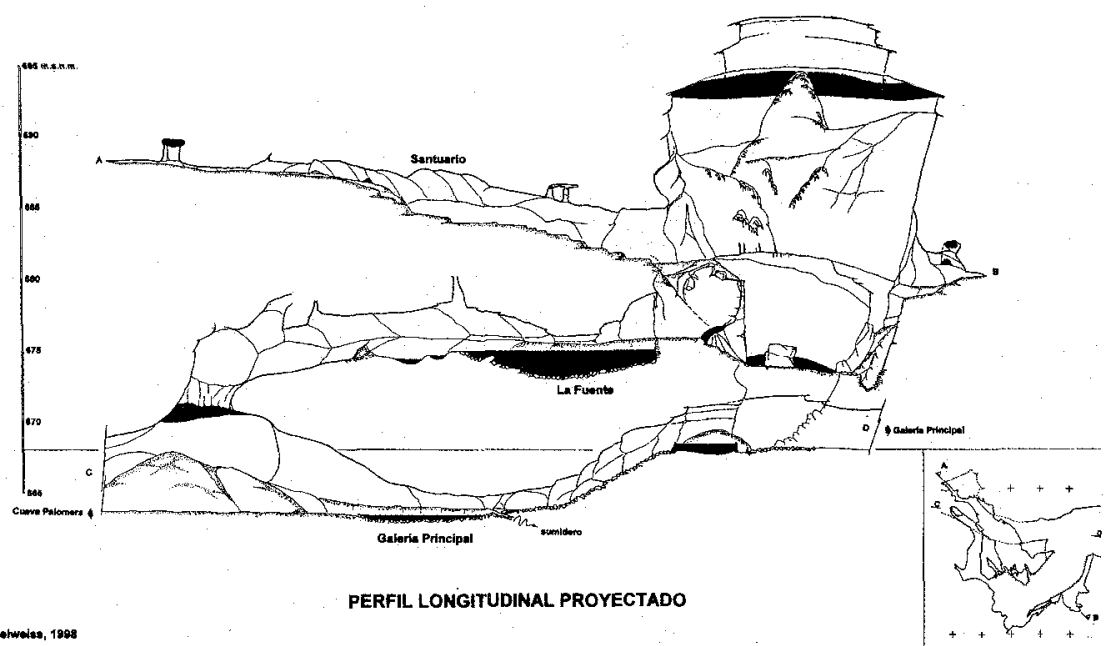

Fig. 4. Perfil proyectado (eje NW-SE) de la Sala de la Fuente.

es el momento aún de valoraciones y conclusiones. Se ha entendido el estudio de la sala que nos ocupa como el punto de partida del proyecto global y, sobre todo, como la propia evaluación de la viabilidad del proyecto.

De alguna manera, la documentación de la Sala de la Fuente está concluida, por lo que el siguiente paso no será otro que entrar de lleno en su estudio; pretendemos llevarlo a cabo próximamente y, una vez concluido, someterlo a la supervisión de la Dirección General de Patrimonio de la Junta de Castilla y León.

Con todo, es preciso que los trabajos realizados en la Sala de la Fuente también se lleven a cabo en las demás galerías con muestras de arte rupestre, de tal manera que, en un futuro, todo el Complejo pudiese ser mostrado e interpretado en su totalidad.

\section{AGRADECIMIENTOS}

El trabajo de campo ha sido financiado por la Junta de Castila y León. Deseamos agradecer la colaboración de Juan Santos González, técnico del Servicio de Delineación de Vías y Obras de la Diputación Provincial de 
Burgos, en el posicionamiento topográfico de los fotogramas tomados para la restitución fotogramétrica de este conjunto. Agradecemos la inestimable colaboración de los miembros del Grupo Espeleológico Edelweiss. Ana Isabel Ortega Martínez disfruta de una Beca de FPI de IBERDROLA en la Universidad de Burgos.

\section{BIBLIOGRAFÍA}

Apellániz Castroviejo, J. M.; UfibarRi Angulo, J. L. (1976): Estudios sobre Atapuerca (Burgos). I. El Santuario de la Galería del Sílex. Cuadernos de Arqueología de Deusto, 5. Bilbao.

Arnálz Alonso, M. A.; Rodríguez Marcos, J. A.; Rojo Guerra, M. A. (1993): «El Arte Prehistórico Burgalés». Historia 16 de Burgos, 5, págs. 51-62.

BÉcares PÉREZ, J. (1987): «Arte Rupestre Prehistórico en la Meseta» en Arte Rupestre en España, págs. 86-95. Revista de Arqueología. Madrid.

Corchón, M. S.; Valladas, H.; Bécares, J.; ARnold, M.; TISnerat, L.; Cachier, H. (1996): «Datación de las pinturas y revisión del arte paleolítico de Cueva Palomera (Ojo Guareña, Burgos, España)». Zephyrus, 49, págs. 37-60.

Fernández Manzano, J.; Romero Carnicero, F. (1994): “Neolítico y Edad de los Metales". Historia del Arte de Castilla y León, I, págs. 25-68. Ed. Ámbito. Valladolid.

Gómez-BARRerA, J. A. (1992): Grabados rupestres postpaleolíticos del Alto Duero. Museo Numantino y Caja Salamanca y Soria. Soria.

Gómez-Bafrera, J. A. (1993): Arte Rupestre Prehistórico en la Meseta Castellano-Leonesa. Junta de Castilla y León. Valladolid.

Grufo EsPeleológico Edelweiss (1986): Monografía sobre el Karst de Ojo Guareña, Kaite, Estudios de Espeleología Burgalesa, 4-5. Excma. Diputación Provincial de Burgos. Burgos.

GuERRA GómEZ, M. (1973): Constantes religiosas europeas y sotoscuevenses (Ojo Guareña, cuna de Castilla). Facultad de Teología. Burgos.

JoRdÁ CERDÁ, F. (1968-1969): «Nuevas representaciones rupestres en Ojo Guareña (Burgos)». Zephyrus, XIX-XX, págs. 61-71.

Llanos VINAA, A.; GaRcía LÁzARO, F. J. (1980a): «Levantamiento fotogramétrico del techo de la sala de las pinturas de la Cueva de Altamira». Altamira Symposium. Ministerio de Cultura, Madrid. págs. 591-611.

Llanos VINAA, A.; GaRcía LÁzARO, F. J. (1980b): «Levantamiento fotogramétrico de Las Cuevas de Altamira». Técnica Topográfica, Madrid.

Moure Romanillo, J. A. (1985): «El Paleolítico y el Arte Rupestre en Burgos» en Historia de Burgos: I. Edad Antigua, Caja de Ahorros Municipal de Burgos, págs. 83-114.

Ortega Martínez, A. I.; Martín Merino, M. A. (1986): "La arqueología del Karst de Ojo Guareña», en Monografía sobre el Karst de Ojo Guareña, Kaite. Estudios de Espeleología Burgalesa, 4-5, págs. 331-389.

Osaba Y Ruiz de EREnCHun, B. (1960): «La arqueología en Ojo Guareña», Revista de Archivos, Bibliotecas y Museos, LXVIII, 1, págs. 177-192.

SAN NICOLÁS DEL TORO, M. (1989): «Trabajos fotogramétricos de objetos cercanos: Arte rupestre en Murcia», Boletín de la Asociación Española de Arte Rupestre, 2, págs. 11-14. 